\title{
Isolation of Coprophilous Fungus Sordaria fimicola from Different Areas of Lahore-Pakistan, and Molecular Characterization by ITS and RAPD- SCAR PCR
}

\author{
Rabia Arif* and Muhammad Saleem \\ Department of Botany, Molecular Genetics Research Laboratory, University of the Punjab, 54590 Lahore, Pakistan \\ *For correspondence: phdgenetics@gmail.com
}

\begin{abstract}
Sixty-one isolates of Sordaria fimicola were isolated from the dung samples of cow (Bos taurus), horse (Equus caballus) buffalo (Bos taurus), donkey (Equus asinus), rabbit (Oryctolagus cuniculus), sheep (Ovis aries), camel (Camelus bactrianus) and goat (Capra hircus) collected from different areas of Lahore by adopting a moist chamber method. The frequency of occurrence $(62.5 \%)$ was higher for horse samples followed by cow and donkey. Microscopic characters such as colour of mycelia mat, shape of perithecia, arrangements of ascospores in the ascus were observed for morphological identification of isolated fungus. ITS region 1 and RAPD-SCAR analyses were made for the molecular characterization of isolates of $S$. fimicola. Diversity indices showed that diversity exists in all isolates. A monomorphic band of 200bp was amplified by ITS1 and ITS2 universal primers in all the isolates and showed $100 \%$ resemblance with $S$. fimicola when introduced in the NCBI BLAST database. Four of eight random decamer RAPD primers after initial screening amplified 22 bands among eight isolates, of which 13(59.0\%) were polymorphic bands with average 5.5 bands per primer. Cluster analysis showed that the similarity coefficients ranged from $0.2-0.6$. RL-4 and RL-7 locus amplified by R3 primer were sent for direct sequencing and contained 485 and 811 bp nucleotides. Successful SCAR primers were developed from sequenced RAPD fragments and submitted to GenBank under the accession number KY098763 for RAPD locus RL-7 and KY120322 for RL-4. Two stable SCAR markers called SCAR 400 and SCAR 500 were developed successfully that can be used to differentiate isolates of different areas. (C) 2017 Friends Science Publishers
\end{abstract}

Keywords: Fungus-isolation; Sordaria fimicola; Herbivore dung; ITS; RAPD-SCAR

\section{Introduction}

Coprophilous fungi are significant constituents that regulate the nutrients in herbivore dung. Coprophilous fungi habitually produce and replicate in a limited range of temperatures, $\mathrm{pH}$, and moisture content and are the excellent source of enzymes and secondary metabolites (André et al., 2011). Ascospores of coprophilous fungi isolated from different dung samples are furthermore helpful to determine the occurrence of ancient herbivores (Ambroise et al., 2013). The genus Sordaria is a unique coprophilous fungus of Sordariaceae family belongs to the class ascomycetes and from the order Sordariales and is closely related to Podospora and Neurospora. All of these fungi nourish or reproduce on herbivore dung, but it can also be isolated from other substrates such as wood, seed or soil in countries where the temperature is high (under warm conditions). All above mentioned genera are widely used in the experimental class of genetics to study crossing over and gene conversion due to the unique arrangement of ascospores in an ascus as genetic model organisms (Ines et al., 2010). Sordaria fimicola is also used, to study the functioning and structure of perithecia, to observe the mechanisms of spore discharge. It is self-fertile (homothallic) and complete its life cycle within 10 days on a large variety of common growth media used in the research laboratories. Environmental factors such as temperature and nutrient resource affect the perithecial development. To grow Sordaria sp. on defined medium it continuously required varied amount of biotin and arginine for the production of perithecium (Webster and Weber, 2007).

Morphological characterization of two species on the basis of morphological and microscopic characters is not authentic and reliable. It is also time consuming, laborious and moreover may lead to wrong identification of species due to different method of handling, harvesting, preparation and storage (Cardoso et al., 2012). Now a day different molecular techniques based upon polymerase chain reaction have been developed to identify or characterise the different species or strains of the same species such as (PCR-RFLP, RAPD, ITS and SCAR markers). These techniques are based on genomic DNA or nucleic acids (Chen et al., 2011).

To cite this paper: Arif, R. and M. Saleem, 2017. Isolation of coprophilous fungus Sordaria fimicola from different areas of Lahore-Pakistan, and molecular characterization by ITS and RAPD-SCAR PCR. Int. J. Agric. Biol., 19: 259-265 
Measuring genetic diversity based upon DNA or nucleotides sequences is reliable as compared to morphological characters because by DNA sequencing one can easily determine the variations in any part of nucleotides (Xu et al., 2010).

During the present study we first time report the use of RAPD-PCR (polymerase chain reaction) to find/detect genetic diversity in different isolates of $S$. fimicola. Problems related to RAPD markers that limit its use (such as sensitivity and reproducibility) to determine the genetic diversity can be overcome by developing stable SCAR markers from sequenced RAPD fragments or amplicons. The general principle is to convert dominant RAPD markers into co-dominant SCAR markers to reduce the limitations. In addition, we developed specific pairs of primers to amplify and find out difference at genetically define loci that have benefits over RAPD markers. These specific markers are called sequence characterized amplified regions. SCARs are considered to be less sensitive and well optimized as compared to RAPDs. SCARs markers are codominant and can amplify more than one allele, which play important role in population and mapping studies.

Very limited studies on the isolation of coprophilous fungi in Pakistan, particularly in Lahore have been done so far. The purpose of this investigation was to isolate the $S$. fimicola from herbivore dung collected from different localities of Lahore Pakistan.

\section{Materials and Methods}

\section{Collecting Sites and Sampling}

Total 125 fresh dung samples of eight different herbivores were collected in zip lock polythene bags with the help of sterile spatula from 7 different areas of Lahore. All samples were labelled with day, date, and animal during summer season (2013) with over a temperature ranges of 35 to $45^{\circ} \mathrm{C}$. Direct isolation of $S$. fimicola from dung samples was performed by moist chamber method described by Hawksworth (1974). To reduce the risk of growth of unwanted organisms during incubation, a minor portion of naphthalene tablet was placed inside the collection bags containing dung samples. To achieve the pure cultures without contamination, grwoth cultures were supplemented with mixture of antibiotics solution (Jacobs et al., 1992). To enhance the perithecial and ascospores development, the ascospores were treated by adopting the method used by $\mathrm{Yu}$ (1954), which used $0.5 \% \mathrm{KOH}$ solution for about $10 \mathrm{~min}$. A desired species was identified from available literature, monographs and mycologist (Lundqvist, 1972). All pure cultures were maintained on dung agar medium (10 g of horse/100 $\mathrm{mL}$ of distilled water).

\section{Molecular Characterization of S. fimicola}

Genomic DNA extraction: All isolates were subjected to g-DNA extraction by employing Pietro et al. (1995) method of DNA extraction. Extracted DNA was quantify using $1 \%$ agarose gel electrophoresis stained with ethidium bromide with size marker (Norgen $1 \mathrm{~Kb}$ ladder) DNA and photographed under gel documentation system (Ugenious3SynGene).

ITS region-1 PCR amplification: $S$. fimicola isolates were exposed to ITS-PCR using ITS1 (TCCGTAGGTGAACCTGCGG) as forward primer and ITS2 (TCCTCCGCTTATTGATATGC) as reverse primer (White et al., 1990). PCR reaction mixture of $25 \mu \mathrm{L}$ contained 10X PCR buffer, 100mM dNTPs (BIOLINE, $\mathrm{UK})$, (25 $\mathrm{mM} \mathrm{MgCl}_{2}$ (BIOLINE), ITS (F+R) primers, template DNA, Taq polymerase (BIOLINE) and $\mathrm{ddH}_{2} \mathrm{O}$ with initial denaturation at $94^{\circ} \mathrm{C}$ for $5 \mathrm{~min}$. and 40 cycles of denaturation (1 $\mathrm{min}$ ) annealing (50.8 temp. for $1 \mathrm{~min}$ ), elongation $\left(72^{\circ} \mathrm{C}\right.$ for $\left.1 \mathrm{~min}\right)$, final elongation $72^{\circ} \mathrm{C}$ for $5 \mathrm{~min}$ and final hold at $4^{\circ} \mathrm{C}$ infinite.

RAPD-PCR amplification: $S$. fimicola isolates were also subjected to RAPD-PCR by 8 RAPD primers for initial screening. To obtain reproducible bands, amplification was carried out in triplicates with optimized conditions and reagents (reaction concentrations and reagents are similar as above) with cycling condition: initial denaturation at $94^{\circ} \mathrm{C}$ for $5 \mathrm{~min}$. and 39 cycles of denaturation (1 min) annealing $\left(35^{\circ} \mathrm{C}\right.$ temp. for $\left.1 \mathrm{~min}\right)$, elongation $\left(72^{\circ} \mathrm{C}\right.$ for $\left.1 \mathrm{~min}\right)$, final elongation $72^{\circ} \mathrm{C}$ for $5 \mathrm{~min}$ and final hold at $4^{\circ} \mathrm{C}$ infinite.

\section{Sequencing of RAPD Fragments}

Two different bright amplified RAPD PCR bands produced from R3 primer were cut from the agarose gel with the help of aseptic scalpel and purified by using mini purification kit and purified bands were sent for direct sequencing to design the primers for SCAR marker to characterize and identification of strain specific marker or locus. By NCBI BLAST, GenBank database online program sequenced RAPD products (http://www.ncbi.nlm.nih.gov/BLAST) were search for sequence homology with other homologous sequence from different species.

\section{Designing and Analysis of SCAR Primers and Markers}

From nucleotide sequence of RAPD fragment, two SCAR primer pairs (F and $\mathrm{R})$ SC-4F (AAGGAACAAAGCCCAGGGTT) SC-4R (ACTTCCTTGTTCGACCGGAC) and SC-5F (TTTGACGGTGCTGAGGACTG) SC-5R (CGGGGATATCATTCGCAGCT) were designed using online Primer3plus (www.bioinformatics.nl/primer3plus/) program. PCR programmed with initial denaturation at $94^{\circ} \mathrm{C}$ for 60 s, followed by 35 cycles of denaturation at $94^{\circ} \mathrm{C}$ for $30 \mathrm{~s}$, annealing at $60^{\circ} \mathrm{C}$ for $1 \mathrm{~min}$, and extension at $72^{\circ} \mathrm{C}$ for $1 \mathrm{~min}$. and final extension at $72^{\circ} \mathrm{C}$ for $5 \mathrm{~min}$. The PCR amplified products were analysed by $1.5 \%$ agarose gel in $0.5 \mathrm{X}$ TAE buffer stained with EtBr at $100 \mathrm{~V}$ for $40 \mathrm{~min}$. 


\section{Results}

\section{Frequency of Occurrence of $S$. fimicola in Different Dung Samples}

Five samples from each site were collected (Table 1). Sixty one isolates $(46.92 \%)$ of Sordaria were extracted. Highest numbers of isolates (13) were isolated from the dung samples collected from Duban Pura area of Lahore (Table 2 ). The maximum numbers of isolates were present in faeces of horse (14 isolates with frequency of occurrence $62.5 \%$ ), followed by cow (12), donkey (11), buffalo (8), goat (5), camel (5), and the lowest number of isolates was found in sheep and rabbit excreta (3).

\section{Morphological Characterization}

To identify the isolated fungus at genus level; morphological characters such as (color of mycelium mat, shape of perithecia, arrangements of ascospores in the ascus) were studied under stereo-microscope (Table 3). No hypha was observed after six, eight and twelve hours, a small hypal tip started to appear after $16 \mathrm{~h}$ under microscope. A cluster of hyaline mycelium in the form of a ring appeared around the dung agar block that can be easily seen with the help of naked eye after $24 \mathrm{~h}$. A thick mass of mycelium appeared after $48 \mathrm{~h}$ that covered the whole petri plates. After one week of incubation mycelia mat turns into greyish green. No perithecia appeared within one week after 10 days small fruiting bodies just like the tip of the common pin appeared on the plates (Fig. 1). All perithecia were surrounded by gelatinous sheath. Due to the presence of sheath it was difficult to separate the perithecia from each other (Fig. 2).

\section{Molecular Characterization}

For molecular identification of the genus at species level PCR amplification of ITS region 1 (rDNA) of the fungal isolates was targeted (Fig. 3). Sequence homology search was performed using BLAST at NCBI database and showed 100 similarities with fimicola sp. of Sordaria.

S. fimicola isolates were subjected to RAPD PCR to determine the genetic relatedness and genetic diversity (Table 4). Four of eight primers showed reproducible bands on $1.5 \%$ agarose gel. Highest polymorphisms was calculated by RAPD primer R-3 i.e., $83.33 \%$, while RAPD primer R-8 determined least genetic diversity (25\%) among the isolated isolates of S. fimicola. R-1 primer amplified four polymorphic bands and two monomorphic bands (Fig. 4).

Based on the RAPD-PCR amplification profiles generated by $\mathrm{R} 3$ primer, a cluster dendrogram was constructed (Fig. 5). Cluster showed that the similarity coefficient ranged between 0.2-0.6. Major cluster divided into two main clusters (I and II). Cluster I further divided into two clusters (A1 and B1). Cluster B1 have two isolates 2 and 4 . A1 cluster further divided into two clusters A2 (have three isolates $3,7,8$ ) and B2 (one isolate i.e., 5). Major cluster II divided into $\mathrm{C} 1$ and $\mathrm{C} 2$ cluster and each cluster contain one isolate.

RAPD fragment RL-4 consisted of 485 nucleotides and RAPD fragment RL-8 comprised of 811 nucleotides. BLAST searches of the RAPD sequences in NCBI GenBank record found no homology for the sequences (Fig. 6).

\section{Discussion}

Identification of different species and strains of similar species by morphological and molecular markers have become routine and significant methods. These methods can help scientists to recognize different dung fungal species and populations. This study first time reports the isolation and identification of 61 isolates of $S$. fimicola from dung samples of herbivore. Mungai et al. (2012) isolated various fungi by adopting same isolating technique from dung samples collected from different areas of Kenya. Mohsin et al. (2011) isolated 87 various strains of temperature resistant cellulolytic fungi from different areas of Pakistan from different substrates including dung of herbivores by adopting the moist chamber method. Mushtaq et al. (2004) isolated five species of yeast from garden soil collected from different areas of Karachi and identified on the basis of morphological characters.

According to Bell and Wheeler (1986) during the first phase of fungal succession on herbivore dung member of Phycomycetes e.g. Mucor and Pilobolus appeared within 3-4 days of incubation and later on Ascobolus e.g. appeared within 6 to 7 days of incubation and in the end member that had flask shaped perithecia appeared usually 9 to 10 days. Appearance of S. fimicola in the last is mainly due to the fact that it required 9-12 days for the development of mature perithecia (Watanabe, 1997; Richardson, 2008), which is confirmed from the present findings.

In numerous ascomycetes, mucilaginous sheath is present as outmost sheet around perithecia e.g. in Ascobolus immersus and S. fimicola (Zhang et al., 2006). Mucilaginous attachments in Sordaria and Podospora help the ascospores to attach the substrate and vegetation firmly and when these ascospores inhaled by other herbivores along with grass then fungal usual life cycle starts (Bell, 2005). Arrangement of ascospores in linear fashion in an ascus and presence of mucilaginous sheath is therefore considered important morphological characters for the identification of $S$. fimicola. Ascospores projectile in ascomycetes especially for coprophilous fungi (Ascobolus immersus and S. fimicola) is an adaptive character and as a result ascospores dispersed to longer distance (Webster and Weber, 2007). 
Table 1: Source of herbivore dung and area of collection of samples

\begin{tabular}{lll}
\hline Animal & Scientific name & Area of Collection \\
\hline Cow & Bos taurus & Duban Pura Multan Rd, River Ravi, Band Rd, China scheme, \\
Horse & Equus caballus & River Ravi, Band Rd. Urdu Bazar, Sabzi mundi Yateem khana, Kalasha Kaku \\
Bufallo & Bos taurus & Band Rd. River Ravi, China Scheme, Kalasha Kaku \\
Donkey & Equus asinus & Kalasha Kaku, Duban Pura, Udru Bazar Lahore, China Scheme \\
Rabbit & Oryctolagus cuniculus & Lahore Zoo, China Scheme \\
Sheep & Ovis aries & Duban pura, Lahore Zoo, \\
Camel & Camelus bactrianus & Lahore Zoo, Duban pura \\
Goat & Capra hircus & China scheme, Duban pura \\
\hline
\end{tabular}

Table 2: Number of Sordaria isolates from different dung samples and areas along with diversity indices where $(\mathrm{H})$ is Shannon diversity index; (D) is Simon's diversity index; F is the Frequency of Occurrence

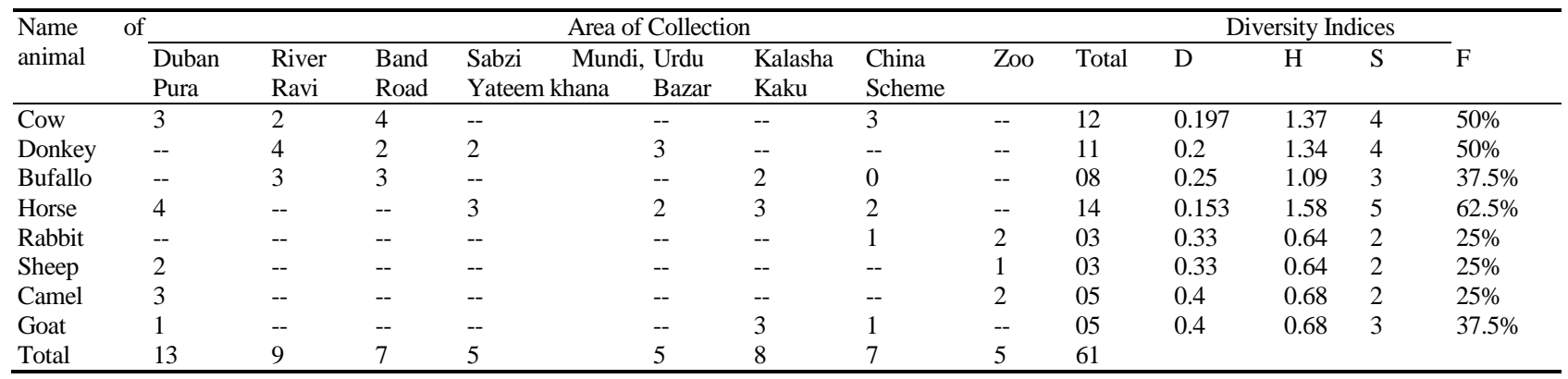

Table 3: Morphological study of S. fimicola under microscope

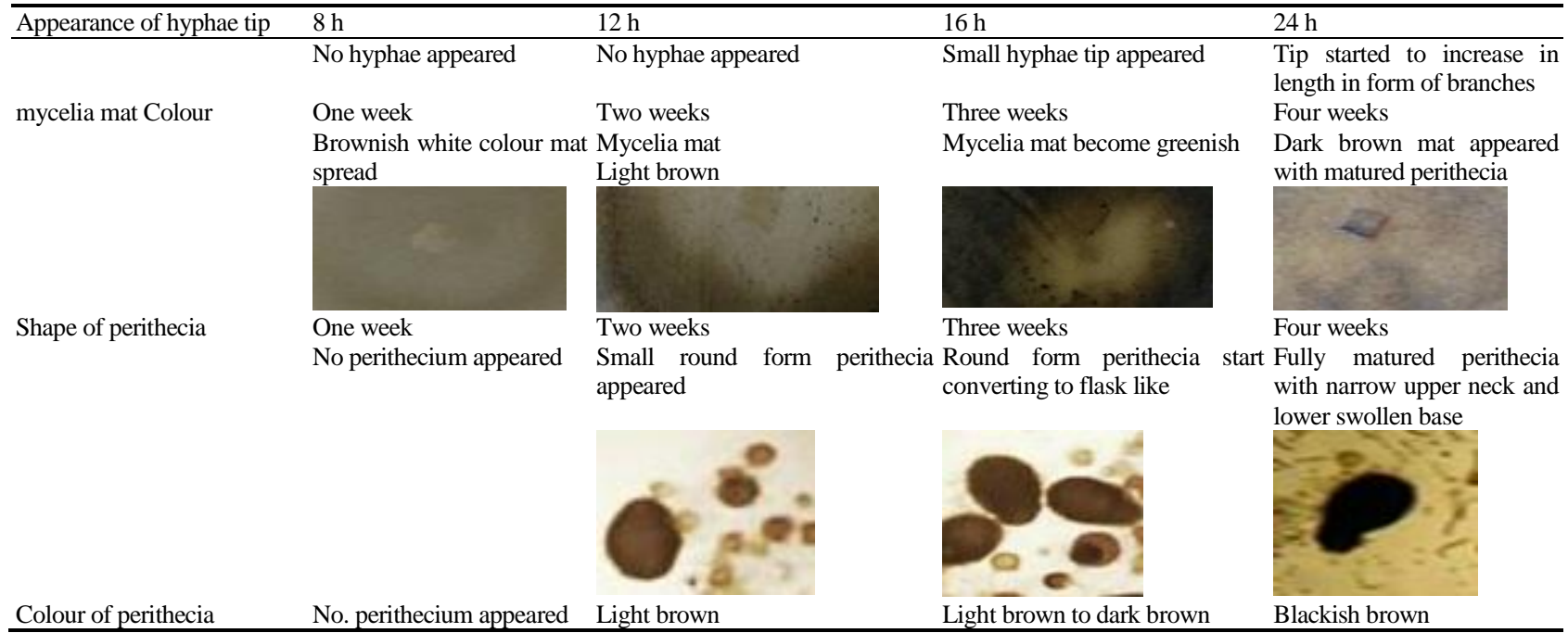

Table 4: List of arbitrary decamer RAPD primer with sequences; TNB = total number bands; TNM = total number of monomorphic band; TNP = total number of polymorphic band; - indicating primers with no amplification and PIC = polymorphic information content

\begin{tabular}{lllllll}
\hline RAPD Primer & Sequence 5-3 & TNB & TNM & TNP & \% polymorphism & PIC \\
\hline R-1 & TCGGGCTGAG & 6 & 2 & 4 & 66.66 & 0.45 \\
R-2 & CAGAGGTCCC & - & - & - & - & - \\
R-3 & AACAGGGGCA & 7 & 1 & 5 & 83.33 & 0.5 \\
R-4 & GGGTAACGCC & 5 & 2 & 3 & 60.00 & 0.4 \\
R-5 & CAGCACCCAC & - & - & - & - & - \\
R-6 & GAGGATCCCT & - & - & - & - & - \\
R-7 & GGAAGCTTGG & - & - & - & - & 0.3 \\
R-8 & TGAGCCTCAC & 4 & 3 & 1 & 25.00 & \\
\hline
\end{tabular}


S. fimicola showed $62.5 \%$ frequency of occurrence in horse dung and least in rabbit, sheep and camel i.e. $25 \%$ while in other herbivore's dung frequency of occurrence ranging from 37.5 to $50 \%$. This showed that horse dung is excellent substrate for the coprophilous fungus. It has mostly been expected that $S$. fimicola live on dung of horses, hares and rabbits, because in these dung samples percentage of cellulose was higher as compared to other herbivore's dung (Kavak, 2012). In the present investigation two isolates were isolated from rabbit dung. In their study, Doveri (2014) isolated 5 isolates of S. fimicola from horse collected from different areas of Italy.

Shannon diversity index $(\mathrm{H})$ and Simon's diversity index (D) was reciprocal to each other. Greater the value of $\mathrm{H}$ greater the chance of occurrence and diversity, while lower the value of (D) greater the chance of isolation and diversity. In the present study samples isolated from horse dung had high value for $(\mathrm{H})$ i.e., 1.58 and low value for (D) i.e., 0.15. Zero value for $(\mathrm{H})$ specifies lowest diversity index and value near about one or greater than one mean maximum diversity and richness (Goulart et al., 2005). Our results showed that all the isolates of $S$. fimicola isolated from different areas were more or less different.

Molecular identification of different fungal species and for measuring genetic differences within same or different populations through ITS region is considered to be very trustworthy or suitable. A monomorphic band of about $200 \mathrm{bp}$ was amplified in all the isolates and no size polymorphism was observed. Sumitha et al. (2015) identified Chichliobolus sp. from the samples collected from infected eyes of different patient by targeting ITS region of isolated fungal sp. The current results revealed identification of isolates of S. fimicola through sequencing of ITS region 1. Ishfaq et al. (2014) found $100 \%$ similarity among ten locally isolated $A$. niger strains when $18 \mathrm{~S}$ rDNA region was targeted. Arif and Saleem (2016) authenticated 6 different strains of $S$. fimicola by targeting full ITS region and also found $100 \%$ resemblance between strains isolated from two opposite slopes of Evolution Canyon, Israel. ITS region is highly conserved within species and sequence homology decreases between different species, or class (Shaw et al., 2002).

To date based on our knowledge no work is done on the diversity analysis of this model fungus isolated from different areas using DNA markers. Our interest was to collect the genotypic data of $S$. fimicola to access the genetic diversity, using RAPD-SCAR PCR, as lot of phenotypic and gene conversion data has already been collected by various workers (Saleem et al., 2001). Improved Randomly amplified polymorphic analysis (RAPD-SCAR-PCR) is one of the important marker, and routine procedure to characterize different species of plant, animals and fungi by different workers.

SCAR markers segregate into single or few alleles instead of multiple alleles, they are more precise as compared to other dominant and co-dominant markers.

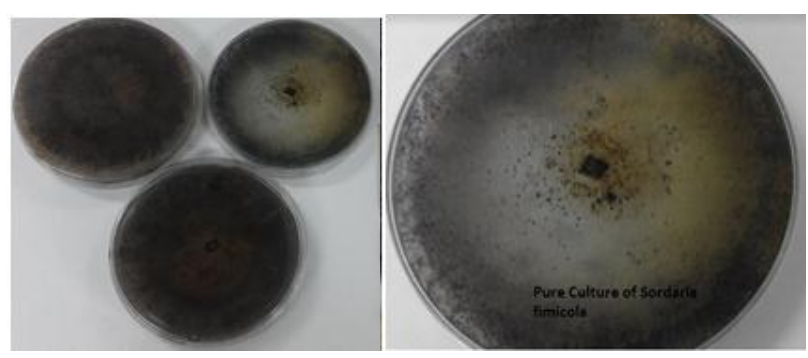

Fig. 1: Pure cultures of $S$. fimicola maintained on Dung Agar medium isolated from different dung of herbivores; Black dots in the figure are mature perithecia can easily be seen with naked eye

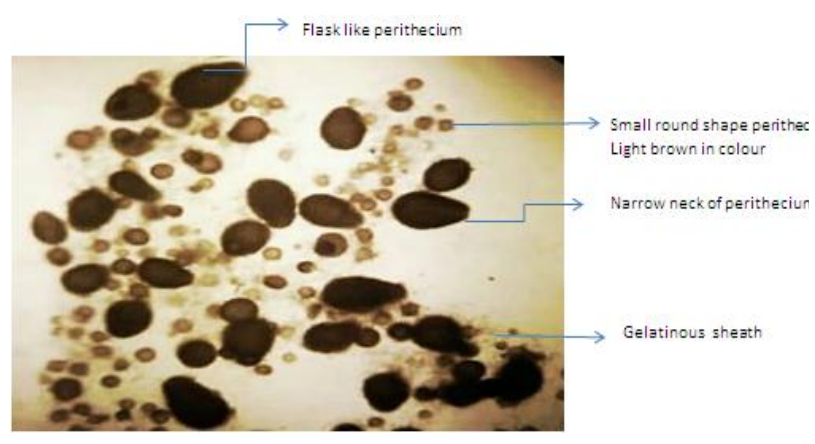

Fig. 2: Different form and shapes of perithecia of $S$. fimicola under microscope

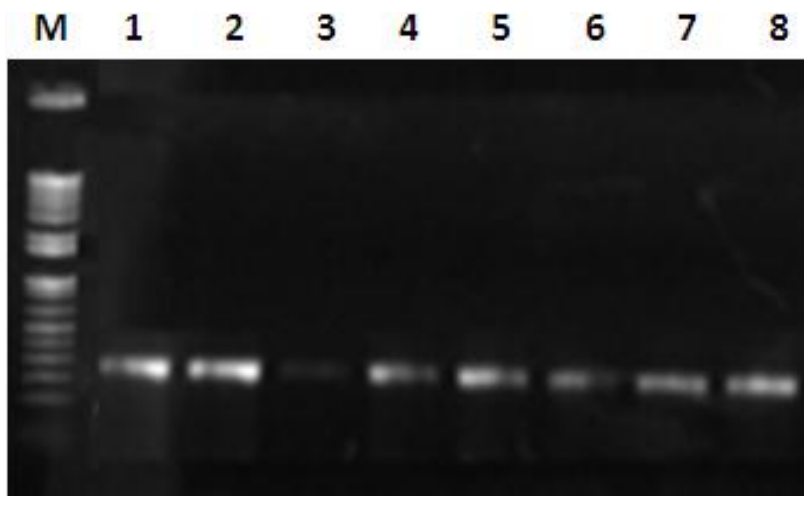

Fig. 3: Amplification of Internal transcribed spacer region 1 by ITS1 and ITS2 primer among different isolates of $S$. fimicola amplifying a monomorphic band of about 200bp in all isolates

The PCR results by SCAR primer SC-4F/SC-4R indicated that the PCR products with expected size were observed in all eight isolates of $S$. fimicola. Specifically, one sample isolated from Sabzi Mandi (Lane 4 in Fig. 7A) showed a weak band of smaller size than expected, which might be a non-specific band. The PCR amplifications with SCAR primer SC-5F/SC-5R amplified the desired size band in isolate 1 and 2, without any amplification in other six $S$. fimicola samples or strains isolated from Duban Pura and River Ravi (Lane 1 and 2, Fig. 7B). 


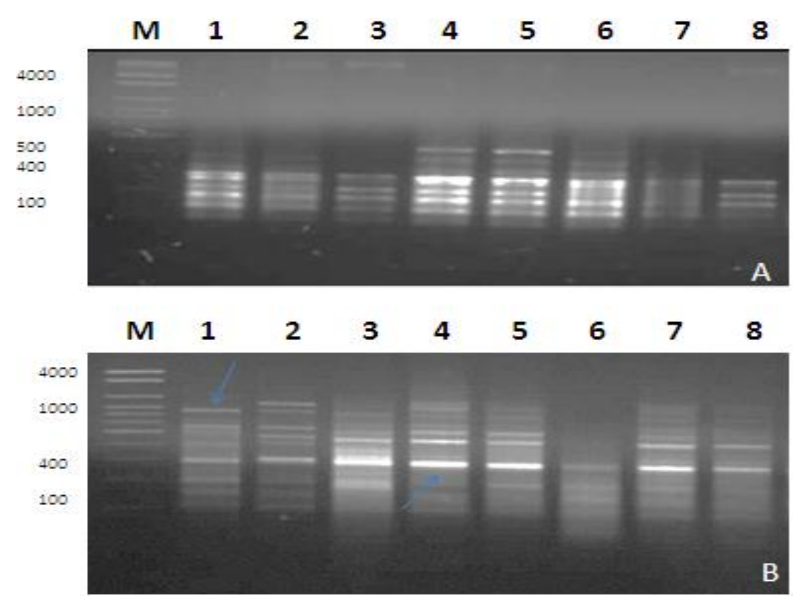

Fig. 4: RAPD profile for S. fimicola. (A) RAPD profile for $S$. fimicola generated by R-1 primer. (B) RAPD profile for $S$. fimicola generated by R-3 primer. Blue arrow indicating the RAPD fragment excised from agarose gel for sequencing to develop SCAR markers

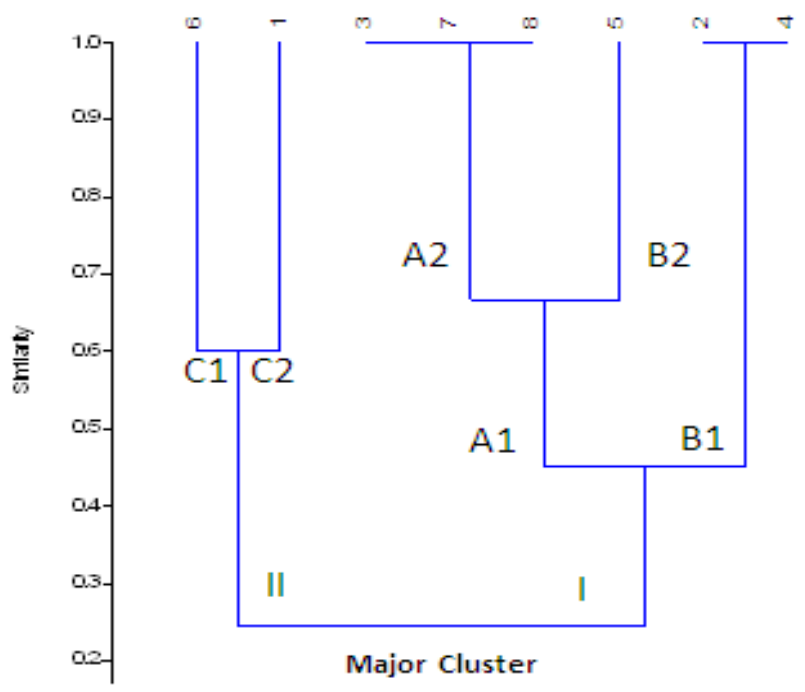

Fig. 5: Cluster analysis of eight isolates of S. fimicola generated by R-3 primer using Paleontological statistics software package for education and data analysis (PAST)

Parsad (2014) employed the same technique to determine the phylogenetics and relatedness among nine different cultivars of Hibiscus rosasinensis using two random primers and calculated the genetic similarity on the bases of two alleles (band absent and band present). Our work is further supported by Jingliang et al. (2015) who established stable SCAR markers for validation of Litchi chinensis from well-developed RAPD and ISSR techniques and no significant sequence homology was found in BLAST search with any other species, same is the case in our study. Some previous studies used this technique for the molecular depiction of plants and other fungi though; this is the first RAPD-SCAR investigation on S. fimicola.

\begin{abstract}
GTAATCACGAATTTCACCTCCCACAACTCAATATTCCCTACAAGT GCTTCCCTTCCTTTI AAGGAACAAAGCCCAGGGTTAGAGTCCGGCAACGGCGTACAGTTCTCCCGGGGGTGCGCG TCTGCTTTGCCCCTCGGGAT TGTCCCTTCAACTTCTGAGCTTTCGATTACTTCATCAACA AGTTTTATTAGGGAGGGTATTGGACTCCCGCTTTGGCTGGCCTTTCATTTAGCTAATTCG AATCTCACTATCGATATCTTGAGGGTTCACCGGTATCCTGAAAGCAACATCTGATAAAAC CAATAAACACGAATCTATCCCTACTTTTCTATCAGCCCGAATTGTACCTCCATATCAGCC CTAATTCAGGCCCAACTCAGGCCAAAACGGACGAAAATCAATGAT GTAAGAGGCCCTCGA GAACCTGCTAAGAT GAAT GTTTTCAACCGAGGTCCGGTCGAACAAGGAAGTAATCTATTT ATAGG

$>\mathrm{b}$

GGCCCCGTGGTGTAGTGAGTGCTTAGTTGCTGTATCTGTCGGGGAGGTGGGGACGTGGTI GAAGTTGTGGTTACGGTAGTACGCATTTTACAGAGTGAGGGGGTTTCTCCTGGTTTGACG GTGCTGAGGACTGGTCGACCCGCTCCGCGGAAT GGACGTCTGT GTCCAGGCATATATTGT GGGTTTTGCCCAGCCGCCGTAGACCATGGGTT GTATATAAACGAT GGAAAATGTATCTGG ATATTAGCTGGACACTCCGCCGCTCTCTTATGCGTGAACAAAGCATCTTCCCCCTTGTTC TCTTGTGATATGCACAGGTTGTATCTGATAAAAGTCCCTCCTACCTCGTCCGCGTCTTTT GAATCGAACATAAATTAAGCTCCAGTTAGTTGCAAGATTGTCTTTTGGCACAATATGAAA TGCACGTTTTTTGTACGACCGTACAGACAAAACTGGCTCACTAGGCGTAGAACAAAGATC AAGAGGGTTGCCATGTCAACGACATGAGATATGCGCAGGTTCACGATTAATGAAGGATGG CTTTTTGGCAAGAACGGGCTTCTGACAGCCAGAAATACCCAGCCCTATGAACTATTCTAC TCCAAGCTGCGAATGATATCCCCGTTTCAGTAGAATATCCCT GAATAATGACGTTCTGCC TCCATACTCAGCCCCGAAGTCCAGAAGAATGCTTCGGACTAGAAAGCACTACATGCCTAC GGAACCCATTGTTTCGTGGGAGCCGCGTTTG
\end{abstract}

Fig. 6: RAPD amplicon nucleotide sequences. (a) The 485 nucleotide sequences of RL-4 fragment. (b) The 811 nucleotide sequences of RL-8 fragment. Under line Bold letter indicating position of SCAR primers forward and reverse
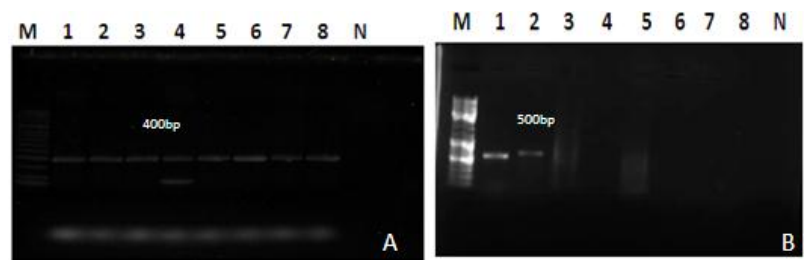

Fig. 7: Development of SCAR markers of $\mathrm{SCAR}_{400}$ and SCAR $_{500}$ from RAPD Locus RL-4 and RL-7 by SCAR primers. (A) SCAR marker $\mathrm{SCAR}_{400}$ by $\mathrm{SCAR}$ primer $\mathrm{SC}$ $4 \mathrm{~F}$ and SC-4R. (B). SCAR marker $\mathrm{SCAR}_{500}$ by SCAR primer SC-7F and SC-7R. Lanes 1-8 indicate isolates of $S$. fimicola listed in Table 2

\section{Conclusion}

The present study illustrated that herbivores dung are the excellent source for $S$. fimicola. We established SCAR markers specific for the confirmation of $S$. fimicola. The results also specify that the improved RAPD analysis has the potential for the genetic analysis of any species. Thus, the combination of the two techniques provides a simple and consistent tool for the genetic categorization of any species.

\section{References}

Ambroise, G.B., A.B. Shonil and J.W. Katherine, 2013. Do dung fungal spores make a good proxy for past distribution of large herbivores? Quat. Sci. Rev., 62: 21-31

André, L.C.M., F.BT. Sandra, M. Elaine, J.P. Paulo and A.Q. Maria, 2011. Zygomycetes from Herbivore Dung in the Ecological Reserve of Dois IrmÃOs, Northeast Brazil. Braz. J. Microbiol., 42: 89-90 
Arif, R. and M. Saleem, 2016. Study of Internal transcribed spacer region-II and full ITS region in different natural strains of Sordaria fimicola. Sci. Int., 28: 2561-2567

Bell, A.A. and M.H. Wheeler, 1986. Biosynthesis and functions of fungal melanins. Annu. Rev. Phytopathol., 24: 411-451

Bell, A., 2005. An Illustrated Guide to the Coprophilous Ascomycetes of Australia. CBS Biodiversity Series No 3, Utrecht, The Netherlands

Cardoso, J.M., L. Fonseca and I. Abrantes, 2012. Direct molecular detection of the pinewood nematode, Bursaphelenchus xylophilus, from pine wood, bark and insect vector. Eur. J. Plant Pathol., 133: 419-425

Chen, H., X. He, J.Pan and J.Zhu, 2011. Advances on Application of Molecular Markers in Longan Researches. Chin. Agric. Sci. Bull., 26: 26-30

Doveri, F., 2014. Coprophilous pyrenomycete from the Tuscan Archipelago and adjacent peninsular coast: description of five species new to Italy. Mycosphere, 5: 188-216

Goulart, M.F., J.P.L. Filho and M.B. Lovato, 2005. Phenological variation within and among populations of Plathymenia reticulate in Brazilian Cerredo, the Atlantic Forest and Transitional Sites. Ann. Bot., 96: 445-455

Hawksworth, D.L., 1974. Mycologist's Handbook. Commonwealth Mycological Institute, Kew, Hawksworth, UK

Ines, E., N. Minou and K. Ulrich, 2010. Sordaria macrospora, a model organism to study fungal cellular development. Eur. J. Cell Biol., 89: 864-872

Ishfaq, M., N. Mahmood, A.N. Idrees and M. Saleem, 2014. Molecular and biochemical screening of local Aspergillus niger strains efficient in catalase and laccase enzyme production. Int. J. Agric. Biol., 16:177-182

Jacobs, R.A., A. Wong-Beringer and B.J. Guglielmo, 1992. Treatment of funguria. JAMA, 267: 2780-2785

Jingliang, C., L. Yan, K.M. Asaduzzaman, W. Chunli, F. Shelly and F. Junjiang, 2015. Development and significance of RAPD-SCAR markers for the identification of Litchi chinensis Sonn. by improved RAPD amplification and molecular cloning. Electr. J. Biotechnol., 18: 35-39

Kavak, H., 2012. Some biological parameters in Sordaria fimicola. Pak. J. Bot., 44: 1079-1082

Lundqvist, N., 1972. Nordic Sordariaceae s. lat. Symb. Bot. Ups., 20: 1-374

Mohsin, M.J., U.H. Ikram, M. Irfana and L. Farooq, 2011. Distribution of cellulolytic-thermophilic fungi on various substrates and geographic locations in Pakistan. Pak. J. Bot., 43: 2621-2625

Mungai, P.G., E. Chukeatirote, J.G. Njogu and K.D. Hyde, 2012. Studies of coprophilous ascomycetes in Kenya: Sordariales from wildlife dung. Mycosphere, 3: 437-448
Mushtaq, M., Sharfun-Nahar and M.H. Hashmi, 2004. Isolation and identification of yeast flora from soil of Karachi, Pakistan. Pak. J. Bot., 36: 173-180

Parsad, M.P., 2014. Molecular characterization and genetic diversity determination of Hibiscus species using RAPD molecular markers. Asian J. Plant Sci. Res., 4: 50-56

Pietro, S., T.M. Fulton, J. Chunwongesm and S.D. Tanksley, 1995. Extraction of high quality DNA for Genome Sequencing. Mol. Biol. Rep., 13: 207

Richardson, M.J., 2008. Coprophilous fungi from the Greek Aegean islands. Mycol. Bal., 5: 23-32

Saleem, M., B.C. Lamb and E. Nevo, 2001. Inherited differences in crossing over and gene conversion frequencies between wild strains of Sordaria fimicola from Evolution Canyon. Genetics, 159: 15731593

Shaw, P.C., J. Wang and P.P.H. But, 2002. Authentication of Chinese Medicinal Materials by DNA Technology, pp: 1-23. World Scientific Publishing Corporation Limited, Tokyo, Japan

Sumitha, J., C. Padmalatha, A.J.A. Ranjit and P. Vanitha, 2015. Genomic profile of a new novel fungal isolates, Cochliobolus $s p$. from human eye infections. Int. J. Pure Appl. Zool., 3: 154-161

Watanabe, T., 1997. Stimulation of perithecium and ascospores production in Sordaria fimicola by Armillaria and various fungal species. Mycol. Res., 101: 1190-1194

Webster, J. and R.W.S. Weber, 2007. Introduction to Fungi, pp: 315-318. Cambridge University Press, Cambridge England

White, T.J., T. Bruns, S. Lee and J.W. Taylor, 1990. Amplification and direct sequencing of fungal ribosomal RNA genes for phylogenetics. In: PCR Protocols: A Guide to Methods and Applications, pp: 315322. Innis, M.A., D. H. Gelfand, J.J. Sninsky and T.J. White (eds.). Academic Press, Inc., New York, USA

Xu, G.W., C.W. Magill, K.F. Schertz and G.E. Hart, 2010. An RFLP linkage map of Sorghum bicolor (L). Theor. Appl. Genet., 89: 139145

Yu, C.C.C., 1954. The Culture and Spore Germination of Ascobolus with Emphasis on A. magnificus. Amer. J. Bot., 41: 21-30

Zhang, N., L.A. Castlebury, A.N. Miller, S.M. Huhndorf and C.L. Schoch, 2006. An overview of the systematics of the Sordariomycetes based on a four-gene phylogeny. Mycologia, 98: 1076-1087

(Received 22 November 2016; Accepted 19 December 2016) 